\title{
The VISCACHA survey
}

\section{Star clusters counterpart of the Magellanic Bridge and Counter-Bridge in 8D*}

\author{
B. Dias ${ }^{1}$, M. S. Angelo ${ }^{2}$, R. A. P. Oliveira ${ }^{3}$, F. Maia ${ }^{4}$, M. C. Parisi ${ }^{5,6}$, B. De Bortoli ${ }^{7,8}$, S. O. Souza ${ }^{3}$, \\ O. J. Katime Santrich ${ }^{9}$, L. P. Bassino ${ }^{7,8}$, B. Barbuy ${ }^{3}$, E. Bica ${ }^{10}$, D. Geisler ${ }^{11,12,13}$, L. Kerber $^{9}$, A. Pérez-Villegas ${ }^{3,14}$,
} B. Quint ${ }^{15}$, D. Sanmartim ${ }^{16}$, J. F. C. Santos Jr. ${ }^{17}$, and P. Westera ${ }^{18}$

${ }^{1}$ Instituto de Alta Investigación, Sede Esmeralda, Universidad de Tarapacá, Av. Luis Emilio Recabarren 2477, Iquique, Chile e-mail: bdiasm@academicos.uta.cl

2 Centro Federal de Educação Tecnológica de Minas Gerais Av. Monsenhor Luiz de Gonzaga, 103, 37250-000 Nepomuceno, MG, Brazil

3 Universidade de São Paulo, IAG, Rua do Matão 1226, Cidade Universitária, São Paulo 05508-900, Brazil

4 Instituto de Física, Universidade Federal do Rio de Janeiro, 21941-972 Rio de Janeiro, RJ, Brazil

5 Observatorio Astronómico, Universidad Nacional de Córdoba, Laprida 854, X5000BGR Córdoba, Argentina

${ }^{6}$ Instituto de Astronomía Teórica y Experimental (CONICET-UNC), Laprida 854, X5000BGR Córdoba, Argentina

${ }^{7}$ Facultad de Ciencias Astronómicas y Geofísicas de la Universidad Nacional de La Plata, and Instituto de Astrofísica de La Plata (CCT La Plata - CONICET, UNLP), Paseo del Bosque s/n, B1900FWA La Plata, Argentina

8 Consejo Nacional de Investigaciones Científicas y Técnicas, Godoy Cruz 2290, C1425FQB Ciudad Autónoma de Buenos Aires, Argentina

9 Departamento de Ciências Exatas e Tecnológicas, UESC, Rodovia Jorge Amado km 16, 45662-900 Ilhéus, Brazil

10 Departamento de Astronomia, IF - UFRGS, Av. Bento Gonçalves 9500, 91501-970 Porto Alegre, Brazil

11 Departamento de Física y Astronomía, Universidad de La Serena, Avenida Juan Cisternas 1200, La Serena, Chile

12 Instituto de Investigación Multidisciplinario en Ciencia y Tecnología, Universidad de La Serena Benavente 980, La Serena, Chile

13 Departmento de Astronomía, Universidad de Concepción, Casilla 160-C, Concepción, Chile

14 Instituto de Astronomía, Universidad Nacional Autonóma de México, Apartado Postal 106, C.P. 22800 Ensenada, Baja California, Mexico

15 Gemini Observatory/NSF's NOIRLab, Casilla 603, La Serena, Chile

16 Carnegie Observatories, Las Campanas Observatory, Casilla 601, La Serena, Chile

17 Departamento de Física, ICEx - UFMG, Av. Antônio Carlos 6627, Belo Horizonte 31270-901, Brazil

18 Universidade Federal do ABC, Centro de Ciências Naturais e Humanas, Avenida dos Estados, 5001, 09210-580 Santo André, Brazil

Received 30 November 2020 / Accepted 26 February 2021

\section{ABSTRACT}

Context. The interactions between the Small and Large Magellanic Clouds (SMC and LMC) created the Magellanic Bridge; a stream of gas and stars pulled out of the SMC towards the LMC about $150 \mathrm{Myr}$ ago. The tidal counterpart of this structure, which should include a trailing arm, has been predicted by models but no compelling observational evidence has confirmed the Counter-Bridge so far.

Aims. The main goal of this work is to find the stellar counterpart of the Magellanic Bridge and Counter-Bridge. We use star clusters in the SMC outskirts as they provide a 6D phase-space vector, age, and metallicity which help characterise the outskirts of the SMC. Methods. Distances, ages, and photometric metallicities were derived from fitting isochrones to the colour-magnitude diagrams from the VISCACHA survey. Radial velocities and spectroscopic metallicities were derived from the spectroscopic follow-up using GMOS in the CaII triplet region.

Results. Among the seven clusters analysed in this work, five belong to the Magellanic Bridge, one belongs to the Counter-Bridge, and the other belongs to the transition region.

Conclusions. The existence of the tidal counterpart of the Magellanic Bridge is evidenced by star clusters. The stellar component of the Magellanic Bridge and Counter-Bridge are confirmed in the SMC outskirts. These results are an important constraint for models that seek to reconstruct the history of the orbit and interactions between the LMC and SMC as well as constrain their future interaction including with the Milky Way.

Key words. Magellanic Clouds - galaxies: star clusters: general - galaxies: evolution 


\section{Introduction}

The past interaction history between the Small and Large Magellanic Clouds (SMC, LMC) and of both galaxies with the Milky Way has been a controversial topic of discussion in the last few decades. A canonical scenario describes the SMC and LMC as bound to the Milky Way for more than $5 \mathrm{Gyr}$, and it prescribes that they only became a pair recently, meaning that the Magellanic Stream and Leading Arm were formed from the interaction among the three galaxies (e.g. Gardiner \& Noguchi 1996; Diaz \& Bekki 2011, 2012). An alternative scenario describes the Magellanic System as a bound SMC-LMC pair that is in its first infall towards the Milky Way or at least in an elongated orbit with a 6 Gyr period (e.g. Besla et al. 2007, 2010). In both cases, simulations are able to roughly reproduce the large-scale gas structure of the Magellanic Stream and Leading Arm, traced by HI gas (e.g. Putman et al. 1998; Nidever et al. 2008), the Magellanic gaseous bridge (e.g. Hindman et al. 1963; Harris 2007), and the old RR Lyrae bridge (e.g. Jacyszyn-Dobrzeniecka et al. 2017; Belokurov et al. 2017). However, the smaller structures still need further simulations to fine-tune the most recent observational constraints. This task is particularly challenging for the complex structure of the SMC that is falling apart with a large line-of-sight depth which is not trivial to characterise (e.g Bekki \& Chiba 2009; Besla et al. 2007; Besla 2011; Dias et al. 2016; Niederhofer et al. 2018; Zivick et al. 2018; De Leo et al. 2020). More multi-dimensional observational constraints are required to help trace the disruption scenario of the SMC.

An important observed feature of the SMC structure that has been detected by different surveys is the large line-ofsight depth of the stellar distribution in the north-eastern SMC, revealing a population at the mean distance of the SMC at about 62 kpc (de Grijs \& Bono 2015; Graczyk et al. 2020) and a foreground population at about $50 \mathrm{kpc}$ (e.g. Nidever et al. 2013; Mackey et al. 2018; Omkumar et al. 2021). The foreground component is interpreted as being the stellar counterpart of the Magellanic Bridge (a.k.a. Bridge), detected only between $2.5^{\circ}$ and $5-6^{\circ}$ from the SMC towards the LMC. If this is a tidal structure, it would be expected that distance correlates with velocity, which is an indication of what was found by the spectroscopic studies of Dobbie et al. (2014) and De Leo et al. (2020). In addition, Pieres et al. (2017) also found a stellar overdensity towards the north of the SMC, which is also interpreted as a tidally stripped structure.

Various simulations are able to reproduce some of the observed features above, but not entirely; they also make predictions that should be further checked with future observations. For example, Diaz \& Bekki (2012) performed $\mathcal{N}$-body simulations, modelling the SMC as a rotating disc (representing the $\mathrm{HI}$ gas) and a non-rotating spheroid (representing the old stellar component). They were able to reproduce the Magellanic Stream and Leading Arm, which formed $\sim 2$ Gyr ago; they also reproduced the Magellanic Bridge (including the bifurcation) and they predicted a tidal counterpart of the Bridge, called the CounterBridge, both formed $~ 150 \mathrm{Myr}$ ago (also predicted by other simulations such as those by Gardiner \& Noguchi 1996 and Besla 2011, although with a different orientation, shape, extension, and density). Diaz \& Bekki (2012) tested three truncation radii to model the spheroid component, the extended model being preferred as it reproduces the morphology and velocity pattern of the Magellanic Bridge better as well as a density break point detected by Nidever et al. (2011) at about $7^{\circ}$ from the SMC centre. This is a remnant of a merger event, although with a simulated position at $\sim 4^{\circ}$. On the other hand, this would imply that the stellar counterpart of the Magellanic Bridge is old, which does not seem to be the case of the exclusively young stellar population detected by Harris (2007), who concluded that the Magellanic Bridge was formed only by gas, whereas stars were formed in situ. In this case, the compact spheroid should be a better choice, which poses a problem for the simulations. Nevertheless, Harris (2007) also found intermediate-age stars in the Bridge, but only in regions closer to the LMC and SMC, and they concluded that these stellar components are bound to their respective galaxies and not tidally stripped, because the old stars are confined in the exponential surface density profile of the LMC, before any break point. They did not analyse, in detail, the SMC Bridge population in the same way, presumably because of the complex geometry of the SMC. Nidever et al. (2011) applied a similar strategy and found a break point in the density profile coincident with the same threshold of the 'pure-bridge' defined by Harris (2007) at about RA $\sim 2.5^{\mathrm{h}}$. Therefore, the stellar counterpart of the Magellanic Bridge (and Counter-Bridge, as a consequence) is indeed a tidally stripped population from the SMC and may be of an old or intermediate age as proposed by the simulations of Diaz \& Bekki (2012).

The old stellar tracers above are usually red clump stars which are useful for overall maps. A more comprehensive picture can be obtained using star clusters as probes, represented by a 6D phase-space vector, age, and metallicity, building up an 8D map of the SMC outskirts. Glatt et al. (2010) derived ages from the colour-magnitude diagrams (CMDs) of 324 SMC clusters, but using shallow photometry from the MCPS survey (Zaritsky et al. 2002), covering only clusters younger than $\sim 1 \mathrm{Gyr}$, and assuming a fixed distance and metallicity. Piatti et al. (2015) analysed 51 Bridge clusters from the VMC survey (Cioni et al. 2011) and found a predominant young population ( 20 Myr), which presumably formed in situ, and an older counterpart $(\sim 1.3 \mathrm{Gyr})$, and they conclude that the Bridge could be older than previously thought. However, they also assumed a fixed distance for all clusters. It remains an open question whether the old population actually corresponds to the foreground tidal stellar counterpart of the Magellanic Bridge. Crowl et al. (2001) derived the 3D positions, ages, and metallicities of 12 SMC clusters and found that the eastern SMC side contains younger and more metal-rich clusters and has a larger line-of-sigh depth than the western side, which is in agreement with other works. They endorsed the use of populous star clusters as probes of the SMC structure.

In this paper we seek to confirm the tidal stripping nature of the SMC Bridge and Counter-Bridge using low-mass star clusters. These clusters have been observed within the VIsible Soar photometry of star Clusters in tApii and Coxi HuguA (VISCACHA) survey ${ }^{1}$ (Maia et al. 2019, hereafter Paper I; Dias et al. 2020) that has been collecting deep and resolved photometric data for star clusters in the outskirts of the Magellanic Clouds, which are more susceptible to be tidally stripped (e.g. Mihos \& Hernquist 1994).

\section{Photometric and spectroscopic data}

The VISCACHA photometric data used here were observed in 2016, 2017, and 2019 under programmes SO2016B-018, SO2017B-014, and SO2019B-019, using the ground-based $4 \mathrm{~m}$ telescope SOAR and its adaptive optics module SAM (Tokovinin et al. 2016). Data reduction, PSF photometry, and CMD decontamination based on membership probability were performed following the methodology presented in Paper I.

The spectroscopic data were observed in 2018 with GMOS, Gemini-S, under the Chile-Brazil-Argentina joint programmes

http://www. astro.iag.usp.br/ viscacha/ 
GS-2018B-Q-208 and GS-2018B-Q-302 to provide radial velocities (RVs), a crucial parameter to characterise tidal tails. The data reduction was done using default tasks of the Gemini data reduction software, automated by a script developed and finetuned by M. S. Angelo ${ }^{2}$. The 1D spectra were extracted, with special attention to the wavelength calibration, that is to say skylines were used as references along with the arc-lamps to enable absolute RV analysis. Proper motions were taken from Gaia Early Data Release 3 (EDR3, Gaia Collaboration 2021a, see Appendix A.2).

\subsection{Statistical isochrone fitting}

The CMD morphology of a star cluster depends on the age, metallicity, distance, and reddening. Therefore, it is mandatory to fit all four parameters together to carry out a self-consistent analysis. We used the SIRIUS code (Souza et al. 2020) that performs a statistical isochrone fitting, with a Bayesian approach based on the Markov chain Monte Carlo sampling method. We adopted the geometrical likelihood function and the membership probability of stars as a uniform prior. The fits are blind to spectroscopic metallicities, which were used to compare with the photometric metallicities as a quality check of both independent analyses.

\subsection{CaT analysis}

Radial velocities were derived by cross-correlation of the observed spectra of individual stars in the clusters against a template synthetic spectrum from the library of Coelho (2014) with typical atmospheric parameters for red giant branch (RGB) stars, that is $\left(T_{\text {eff }}, \log (g),[\mathrm{Fe} / \mathrm{H}],[\alpha / \mathrm{Fe}]\right)=(5000 \mathrm{~K}, 1.0 \mathrm{dex},-1.0 \mathrm{dex}$, 0.4 dex). RVs and uncertainties were derived using fxcor at IRAF, and the conversion from the observed velocities to $\mathrm{RV}_{\text {hel }}$ was calculated using rvcorrect at IRAF (Table 1). Spectroscopic metallicities were derived following the recipes of Dias \& Parisi (2020), using a calibration of the reduced equivalent width of CaII triplet lines (CaT) with metallicity.

The membership selection follows the methodology by Parisi et al. (2009). Briefly, stars within the cluster radius with an RV and $[\mathrm{Fe} / \mathrm{H}]$ within windows of $10 \mathrm{~km} \mathrm{~s}^{-1}$ and $0.2 \mathrm{dex}$ around the group of innermost stars with a common RV and $[\mathrm{Fe} / \mathrm{H}]$ are considered as members. The cluster radii were taken from Santos et al. (2020), whenever available, or from Bica et al. (2020) otherwise.

\section{Discussion}

The starting point to discuss the SMC tidal tails is to argue whether a particular group of star clusters is bound or unbound to the SMC. This is not trivial; however, Nidever et al. (2011) and Diaz \& Bekki (2012) have argued that a sharp break in the radial SMC stellar density distribution indicates tidal distortions. Dias et al. (2014; 2016, hereafter D14,D16) divided the projected distribution of the SMC star cluster population that is outside the SMC main body $\left(a>2^{\circ}\right)$ into three regions (split by voids in the azimuth direction) possibly related to different disrupting episodes: Wing and Bridge, Counter-Bridge, and West Halo. We show in Fig. 1 that all regions share a similar radial density profile, with a clear break point at $a=3.4_{-0.6}^{\circ+1.0}$, which is consistent with (i) the SMC tidal radius $r_{\mathrm{t}}^{\mathrm{SMC}} \sim 4.5^{\circ}$ and the break point for young and old populations found by

\footnotetext{
2 http://drforum.gemini.edu/topic/ gmos-mos-guidelines-part-1/
}

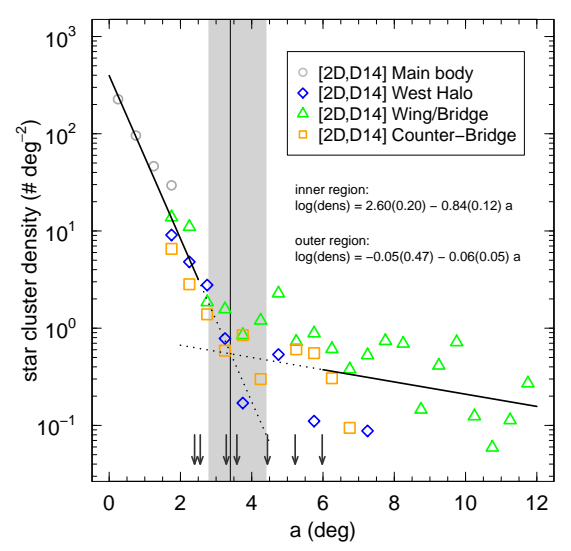

Fig. 1. SMC star cluster radial density profile (catalogued by Bica et al. 2020), using the semi-major axis $a$ of ellipses drawn around the SMC centre as defined by D14 (see also Fig. 2), with $0.5^{\circ}$ bins. Solid lines are the fits to each region, whereas the dotted lines are extrapolation to find the intersection at $a=3.4_{-0.6}^{+1.0}$, represented by the shaded grey $1 \sigma$ area. The arrows indicate the position of the seven clusters.

Massana et al. (2020), (ii) the break point by the simulations of Diaz \& Bekki (2012), and (iii) with the vertex of the $V$ inversion in the metallicity gradient (Parisi et al. 2015; Bica et al. 2020). All seven clusters analysed in this paper are located around the break point or beyond it (see Table 1).

The SMC boundary along the line-of-sight defined by the simulation results of Diaz \& Bekki (2012) is between $\sim 59 \mathrm{kpc}$ and $\sim 65 \mathrm{kpc}$, which we adopted here as a first approximation to identify five Bridge clusters and one (IC 1708) Counter-Bridge cluster (see Table 1 and Fig. 2). The seventh cluster (Bruck 168) has a line-of-sight distance consistent with being bound to the SMC; therefore, we placed this cluster in a transition region. It is worth noting that the spheroid component of the CounterBridge (a.k.a. stars) is much less pronounced and less dense than the respective disc component (a.k.a. gas, see Omkumar et al. 2021); as a result, it is not a surprise to find only a single representation of the Counter-Bridge in our cluster sample. Moreover, there seems to be a trend in the sense that north-eastern Bridge clusters are also closer to us (see Fig. 2), which supports the scenario that the foreground stellar population shapes the start of the Magellanic Bridge towards the LMC.

Another crucial perspective is to analyse how these star clusters move with respect to the distance from the SMC in 3D space. Figure 3 shows how the RVs from the GMOS spectra and Gaia EDR3 proper motions vary with the line-of-sight distance and projected distance on sky $a$. Tidally stripped structures show a distance-velocity correlation, which characterises the Bridge and Counter-Bridge gas and stars very clearly (Gardiner \& Noguchi 1996, Fig. 11; Diaz \& Bekki 2012, Figs. 9 and 15). In Fig. 3, we find a slope between $\mathrm{RV}_{\text {hel }}$ and the line-of-sight distance of $3.7 \pm 1.8 \mathrm{~km} \mathrm{~s}^{-1} \mathrm{kpc}^{-1}$, which is in good agreement with the slope of $3.4 \mathrm{~km} \mathrm{~s}^{-1} \mathrm{kpc}^{-1}$ found by Gardiner \& Noguchi (1996) in their simulations and $4.0 \mathrm{~km} \mathrm{~s}^{-1} \mathrm{kpc}^{-1}$ found by Mathewson et al. (1988) based on Cepheids. This agreement is interesting because Cepheids trace the younger stellar populations, which would support a scenario where Bridge stars were formed in situ. However, our Bridge and Counter-Bridge sample is composed of clusters with ages ranging from $\sim 1-4 \mathrm{Gyr}$, meaning that these clusters were formed before the LMC-SMC encounter $\sim 150 \mathrm{Myr}$ ago, which moved them from their original SMC orbits. Furthermore, Hatzidimitriou et al. (1993) found a slope of $8 \mathrm{~km} \mathrm{~s}^{-1} \mathrm{kpc}^{-1}$ based on red clump stars closer than $60 \mathrm{kpc}$, which would trace intermediate-age and older populations. 
Table 1. Derived parameters for the star clusters.

\begin{tabular}{|c|c|c|c|c|c|c|c|c|c|c|c|}
\hline Cluster & (Gyr) & $\begin{array}{c}{[\mathrm{Fe} / \mathrm{H}]_{\mathrm{CMD}}} \\
(\mathrm{dex})\end{array}$ & $\begin{array}{c}{[\mathrm{Fe} / \mathrm{H}]_{\mathrm{CaT}}} \\
\pm \text { unc.(std.dev.) } \\
\text { (dex) }\end{array}$ & $\begin{array}{c}E(B-V) \\
(\mathrm{mag})\end{array}$ & $(\mathrm{kpc})$ & (deg) & $\begin{array}{c}\alpha_{J 2000} \\
\text { hh:mm:ss }\end{array}$ & $\begin{array}{c}\delta_{J 2000} \\
\mathrm{dd}: \mathrm{mm}: \mathrm{ss}\end{array}$ & $\begin{array}{c}\mathrm{RV}_{\text {hel }} \\
\pm \text { unc.(std.dev.) } \\
\left(\mathrm{km} \mathrm{s}^{-1}\right)\end{array}$ & $\begin{array}{c}\mu_{\alpha} \cdot \cos (\delta) \\
\pm \text { unc.(std.dev.) } \\
\left(\text { mas yr }{ }^{-1}\right)\end{array}$ & $\begin{array}{c}\mu_{\delta} \\
\pm \text { unc.(std.dev.) } \\
\left(\text { mas yr }^{-1}\right)\end{array}$ \\
\hline \multicolumn{12}{|c|}{ Magellanic Bridge } \\
\hline BS 196 & $3.89_{-0.50}^{+0.68}$ & $-0.75_{-0.19}^{+0.22}$ & $-0.89 \pm 0.04(0.08)$ & $0.05_{-0.04}^{+0.04}$ & $50.1_{-2.2}^{+1.6}$ & 5.978 & 01:48:01.8 & $-70: 0$ & $135.5 \pm 1.4$ & $1.12 \pm 0$ & $-1.14 \pm 0.0$ \\
\hline BS 18 & $2_{-0.20}^{+0.22}$ & $0.58_{-0.13}^{+0.13}$ & $.94 \pm 0.06(0.13)$ & $00_{-0.00}^{+0.03}$ & $7_{-3.1}^{+3.0}$ & 4.441 & 01:35:10.9 & $-71: 44: 11$ & $120.3 \pm 3.5(7.9)$ & $1.25 \pm 0.08(0.23)$ & $-1.35 \pm 0.07(0.04)$ \\
\hline HW $56^{(* *)}$ & $3.09_{-0.14}^{+0.22}$ & $-0.54_{-0.12}^{+0.07}$ & $-0.97 \pm 0.12(0.20)$ & $0.03_{-0.02}^{+0.02}$ & $53.5_{-1.2}^{+1.2}$ & 2.397 & 01:07:41.1 & $-70: 56: 06$ & $157.7 \pm 5.4(9.3)$ & $0.99 \pm 0.11(0.04)$ & $-1.27 \pm 0.10(0.29)$ \\
\hline HW 85 & $1.74_{-0.12}^{+0.08}$ & $-0.83_{-0.05}^{+0.07}$ & $-0.82 \pm 0.06(0.14)$ & $0.04_{-0.02}^{+0.02}$ & $54.0_{-2.0}^{+1.2}$ & 5.219 & $01: 42: 28.0$ & $-71: 16: 44$ & $143.2 \pm 3.0(6.8)$ & $1.26 \pm 0.09(0.47)$ & $-1.41 \pm 0.09(0.26)$ \\
\hline Lindsay 100 & $16_{-0.14}^{+0.15}$ & $-0.73_{-0.03}^{+0.03}$ & $0.89 \pm 0.06(0.14)$ & $0.01_{-0.01}^{+0.01}$ & $58.6_{-0.5}^{+0.8}$ & 2.556 & 01:18:16.9 & $-72: 00: 06$ & $145.8 \pm 1.4(3.3)$ & $0.98 \pm 0.05(0.15)$ & $-1.10 \pm 0.05(0.11)$ \\
\hline \multicolumn{12}{|c|}{ Transition } \\
\hline Bruck 168 & $6.6_{-0.9}^{+0.8}$ & $-1.22_{-0.15}^{+0.20}$ & $1.08 \pm 0.06(0.09)$ & $0.00_{-0.01}^{+0.02}$ & $61.9_{-2.0}^{+2.3}$ & 3.584 & $01: 26: 42.7$ & $-70: 47: 01$ & $141.7 \pm 4.6(7.9)$ & $0.94 \pm 0.09(0.08)$ & $-1.15 \pm 0.09(0.04)$ \\
\hline \multicolumn{12}{|c|}{ Counter-Bridge } \\
\hline IC 1708 & $0.93_{-0.04}^{+0.16}$ & $-1.02_{-0.10}^{+0.05}$ & $-1.11 \pm 0.06(0.17)$ & $0.06_{-0.02}^{+0.02}$ & $65.2_{-1.8}^{+1.2}$ & 3.286 & 01:24:55.9 & $-71: 11: 04$ & $214.9 \pm 2.7(6.6)$ & $0.39 \pm 0.10(0.35)$ & $-1.26 \pm 0.07(0.20)$ \\
\hline
\end{tabular}

Notes. Age, $[\mathrm{Fe} / \mathrm{H}]_{\mathrm{CMD}}, E(B-V)$, distance from VISCACHA CMD isochrone fitting, $[\mathrm{Fe} / \mathrm{H}]_{\mathrm{CaT}}, \mathrm{RV}_{\mathrm{hel}}$ from GMOS spectra, $\mu_{\alpha}$, and $\mu_{\delta}$ from Gaia EDR3. Distance $a$ follows the definition by Dias et al. (2014), and $(\alpha, \delta)$ coordinates are from Bica et al. (2020). ${ }^{(*)}$ BS 188 was observed under suboptimal weather conditions, and the resulting isochrone fitting is very sensitive to a handful of stars from the shallow CMD, which could explain the metallicity difference between photometric and spectroscopic $[\mathrm{Fe} / \mathrm{H}]$ (see Appendix A.1). ${ }^{(* *)} \mathrm{HW} 56$ is immersed in a dense field $\left(a=2.397^{\circ}\right.$, the smallest in our sample) that has a very similar CMD as the cluster region; therefore, the statistical decontamination left low-probable member stars on the fainter main sequence region, which may bias the photometric metallicities (see Appendix A.1). These uncertainties do not change the conclusions of this work.

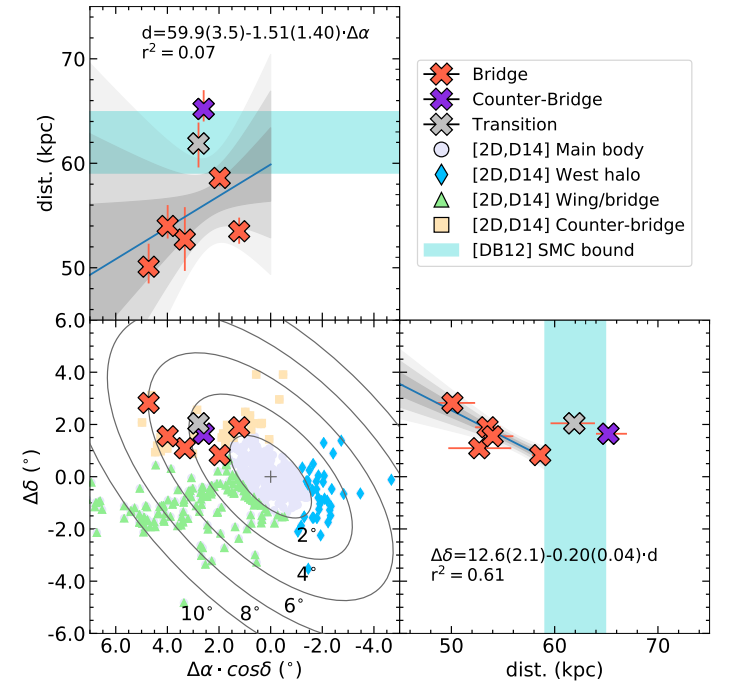

Fig. 2. Position of the seven star clusters in relative equatorial coordinates as defined by $\mathrm{D} 14$, as seen in the planes $(\Delta \alpha, \Delta \delta),(\Delta \alpha$, dist $)$, and $(\Delta \delta$,dist). Background points are all star clusters from Bica et al. (2020). The seven clusters are located in the proposed 'Counter-Bridge' $2 \mathrm{D}$ projected region by $\mathrm{D} 14$, but we now show that this region actually contains Bridge and Counter-Bridge clusters in 6D phase-space. The turquoise shaded area is a first approximation for the region bound to the SMC. Linear fits were performed for Bridge clusters only, considering the uncertainties. See Appendix A.3 for a 3D view of the clusters and a movie is also provided online.

A larger sample of clusters is required to compare the correlation with this study. The correlation of $\mathrm{RV}_{\text {hel }}$ with the projected distance $a$ also reveals a trend in the sense that the Bridge clusters that are farther away from the SMC projected centre are also closer to us and dragging behind towards the LMC. These results are consistent again with one branch of the Magellanic Bridge starting in the north-eastern SMC. The Counter-Bridge also starts in the same region, but so far we only have one point, and a larger sample would help constrain this tidal tail. Gaia EDR3 proper motions show relatively large error bars (Fig. 3); nevertheless, the bulk of Bridge clusters are indeed separated from the Counter-Bridge cluster in $\mu_{\alpha}$, whereas $\mu_{\delta}$ is roughly

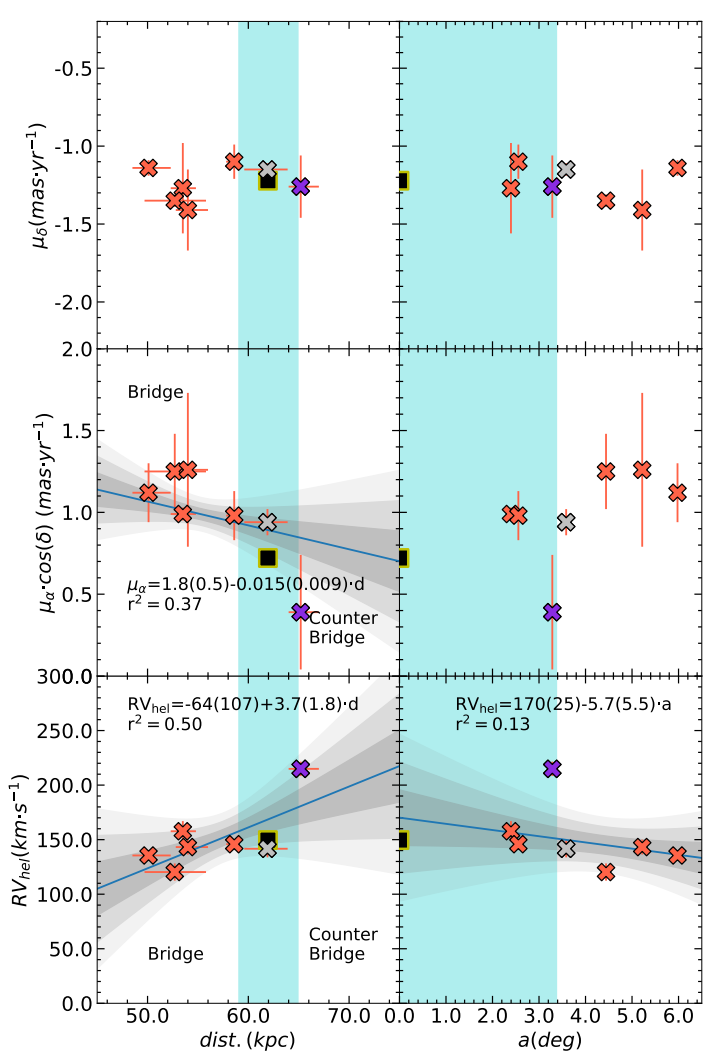

Fig. 3. 3D motion of the seven star clusters. Point colours and shaded areas are the same as in Fig. 2. The black square marks the SMC systemic motion and position (see Table A.1). Linear fits were performed considering the uncertainties and using all clusters to allow direct comparison with other studies (see text). See Appendix A.3 for a 3D view of the clusters and a movie is also provided online.

constant. This is consistent with the scrutiny of Omkumar et al. (2021) on the simulations by Diaz \& Bekki (2012) and also consistent with the Gaia EDR3 proper motion distribution of the Magellanic System (see Appendix A.2).

Last but not least, concerning the eighth dimension analysed here, we report that the metallicity of the five Bridge 
clusters $\left(\left\langle[\mathrm{Fe} / \mathrm{H}]_{\mathrm{CMD}}^{\text {Bridge }}\right\rangle=-0.69 \pm 0.12 \mathrm{dex} ;\left\langle[\mathrm{Fe} / \mathrm{H}]_{\mathrm{CaT}}^{\text {Bridge }}\right\rangle=\right.$ $-0.90 \pm 0.06 \mathrm{dex})$ is $\sim 0.2-0.3 \mathrm{dex}$, that is, $\sim 2 \sigma$ higher than the Counter-Bridge cluster $\left([\mathrm{Fe} / \mathrm{H}]_{\mathrm{CMD}}^{\mathrm{C} \text { - }- \text { Bridge }}=-1.02_{-0.10}^{+0.05} \mathrm{dex}\right.$, $\left.[\mathrm{Fe} / \mathrm{H}]_{\mathrm{CaT}}^{\mathrm{C} .- \text { Bridge }}=-1.11 \pm 0.06 \pm 0.17 \mathrm{dex}\right)$. Moreover, the Bridge clusters are all older than the Counter-Bridge cluster. This suggests differences in metallicities per SMC region as indicated by Crowl et al. (2001) and endorses further investigation. A possible metallicity gradient would not eliminate this difference because more distant Counter-Bridge clusters would have similar metallicities (Parisi et al. 2015) or be even more metal-poor (D14).

\section{Conclusions}

The gaseous complex structure of the Magellanic System and its stellar counterpart, in particular around the SMC, has been observed and simulated by many previous works. We present an analysis of this question using star clusters observed within the VISCACHA survey. The advantage of this approach is that we are able to describe the Magellanic Bridge and CounterBridge in the 6D phase-space plus age and metallicity. We have reached $\sim 1-6 \%$ precision in distance, $\sim 0.5-8 \%$ precision in the mean $\mathrm{RV}$, not to mention $\sim 4-20 \%$ precision in age, and $\sim 0.03-0.22$ dex and $\sim 0.04-0.12$ dex precision in the mean photometric and spectroscopic metallicities.

From the seven clusters analysed here, we found that five belong to the Magellanic Bridge, while one belongs to the Counter-Bridge and another is located at an intermediate region between the two tidal tails. Six-dimensional phase-space vectors of these clusters are consistent with the predictions from the simulations by Diaz \& Bekki (2012), which confirms their unbound current situation. These clusters are 1-4 Gyr old, therefore, they were formed before the SMC-LMC close encounter that generated the SMC tidal tails and moved these clusters away from the SMC main body.

The 2D projected SMC Counter-Bridge region as defined by D14,D16 contains a mix of Bridge and Counter-Bridge clusters in a five-to-one ratio. Consequently, the Magellanic Bridge also has a branch starting from the north-eastern SMC, in addition to the eastern SMC wing and south-eastern RR Lyrae bridge.

The present sample gives important hints on a likely scenario for the formation of a structure in the clouds, in particular the Bridge and the Counter-Bridge. A larger sample is expected to further constrain the perturbed outskirts of the SMC.

Acknowledgements. B.D. and M.C. Parisi acknowledge S. Vasquez for providing his code to measure CaT equivalent widths. Based on observations obtained at the Southern Astrophysical Research (SOAR) telescope, which is a joint project of the Ministério da Ciência, Tecnologia, e Inovação (MCTI) da República Federativa do Brasil, the U.S. National Optical Astronomy Observatory (NOAO), the University of North Carolina at Chapel Hill (UNC), and Michigan State University (MSU). Based on observations obtained at the international Gemini Observatory, a programme of NSF's NOIRLab, which is managed by the Association of Universities for Research in Astronomy (AURA) under a cooperative agreement with the National Science Foundation, on behalf of the Gemini Observatory partnership: the National Science Foundation (United States), National Research Council (Canada), Agencia Nacional de Investigación y Desarrollo (Chile), Ministerio de Ciencia, Tecnología e Innovación (Argentina), Ministério da Ciência, Tecnologia, Inovações e Comunicações (Brazil), and Korea Astronomy and Space Science Institute (Republic of Korea). Programme ID: GS-2018B-Q-208, GS-2018B-Q-302. This work has made use of data from the European Space Agency (ESA) mission Gaia (https: //www . cosmos.esa.int/gaia), processed by the Gaia Data Processing and Analysis Consortium (DPAC, https://www.cosmos.esa.int/web/gaia/dpac/ consortium). Funding for the DPAC has been provided by national institutions, in particular the institutions participating in the Gaia Multilateral Agreement. This research was partially supported by the Argentinian institutions CONICET, SECYT (Universidad Nacional de Córdoba), Universidad Nacional de La Plata and Agencia Nacional de Promoción Científica y Tecnológica (ANPCyT). This study was financed in part by the Coordenação de Aperfeiçoamento de Pessoal de Nível Superior - Brasil (CAPES) - Finance Code 001. A.P.V. and S.O.S. acknowledge the DGAPA-PAPIIT grant IG100319. D.G. gratefully acknowledges support from the Chilean Centro de Excelencia en Astrofísica y Tecnologías Afines (CATA) BASAL grant AFB-170002. D.G. also acknowledges financial support from the Dirección de Investigación y Desarrollo de la Universidad de La Serena through the Programa de Incentivo a la Investigación de Académicos (PIA-DIDULS). R.A.P.O. and S.O.S. acknowledge the FAPESP PhD fellowships nos. 2018/22181-0 and 2018/22044-3. The authors thank the referee for the comments that improved this Letter.

\section{References}

Bekki, K., \& Chiba, M. 2009, PASA, 26, 48

Belokurov, V., Erkal, D., Deason, A. J., et al. 2017, MNRAS, 466, 4711 Besla, G. 2011, Ph.D. Thesis, Harvard University, USA

Besla, G., Kallivayalil, N., Hernquist, L., et al. 2007, ApJ, 668, 949 Besla, G., Kallivayalil, N., Hernquist, L., et al. 2010, ApJ, 721, L97 Bica, E., Santiago, B., Bonatto, C., et al. 2015, MNRAS, 453, 3190 Bica, E., Westera, P., Kerber, L. D. O., et al. 2020, AJ, 159, 82 Cioni, M. R. L., Clementini, G., Girardi, L., et al. 2011, A\&A, 527, A116 Coelho, P. R. T. 2014, MNRAS, 440, 1027

Crowl, H. H., Sarajedini, A., Piatti, A. E., et al. 2001, AJ, 122, 220 de Grijs, R., \& Bono, G. 2015, AJ, 149, 179

de Grijs, R., Wicker, J. E., \& Bono, G. 2014, AJ, 147, 122

De Leo, M., Carrera, R., Noël, N. E. D., et al. 2020, MNRAS, 495, 98

Dias, B., \& Parisi, M. C. 2020, A\&A, 642, A197

Dias, B., Kerber, L. O., Barbuy, B., et al. 2014, A\&A, 561, A106

Dias, B., Kerber, L., Barbuy, B., Bica, E., \& Ortolani, S. 2016, A\&A, 591, A11

Dias, B., Maia, F., Kerber, L., et al. 2020, IAU Symp., 351, 89

Diaz, J., \& Bekki, K. 2011, MNRAS, 413, 2015

Diaz, J. D., \& Bekki, K. 2012, ApJ, 750, 36

Dobbie, P. D., Cole, A. A., Subramaniam, A., \& Keller, S. 2014, MNRAS, 442, 1663

Gaia Collaboration (Brown, A. G. A., et al.) 2021a, A\&A, in press, https: //doi.org/10.1051/0004-6361/202039657

Gaia Collaboration (Luri, X., et al.) 2021b, A\&A, in press, https: //doi .org/ 10.1051/0004-6361/202039588

Gardiner, L. T., \& Noguchi, M. 1996, MNRAS, 278, 191

Glatt, K., Grebel, E. K., \& Koch, A. 2010, A\&A, 517, A50

Graczyk, D., Pietrzynski, G., Thompson, I. B., et al. 2020, ApJ, 904, 13

Harris, J. 2007, ApJ, 658, 345

Hatzidimitriou, D., Cannon, R. D., \& Hawkins, M. R. S. 1993, MNRAS, 261, 873

Hindman, J. V., Kerr, F. J., \& McGee, R. X. 1963, Aust. J. Phys., 16, 570

Jacyszyn-Dobrzeniecka, A. M., Skowron, D. M., Mróz, P., et al. 2017, Acta Astron., 67, 1

Kallivayalil, N., van der Marel, R. P., Besla, G., Anderson, J., \& Alcock, C. 2013, ApJ, 764, 161

Mackey, D., Koposov, S., Da Costa, G., et al. 2018, ApJ, 858, L21

Maia, F. F. S., Dias, B., Santos, J. F. C., et al. 2019, MNRAS, 484, 5702

Massana, P., Noël, N. E. D., Nidever, D. L., et al. 2020, MNRAS, 498, 1034

Mathewson, D. S., Ford, V. L., \& Visvanathan, N. 1988, ApJ, 333, 617

Mihos, J. C., \& Hernquist, L. 1994, ApJ, 425, L13

Nidever, D. L., Majewski, S. R., \& Butler Burton, W. 2008, ApJ, 679, 432

Nidever, D. L., Majewski, S. R., Muñoz, R. R., et al. 2011, ApJ, 733, L10

Nidever, D. L., Monachesi, A., Bell, E. F., et al. 2013, ApJ, 779, 145

Niederhofer, F., Cioni, M. R. L., Rubele, S., et al. 2018, A\&A, 613, L8

Omkumar, A. O., Subramanian, S., Niederhofer, F., et al. 2021, MNRAS, 500, 2757

Parisi, M. C., Grocholski, A. J., Geisler, D., Sarajedini, A., \& Clariá, J. J. 2009, AJ, 138, 517

Parisi, M. C., Geisler, D., Clariá, J. J., et al. 2015, AJ, 149, 154

Piatti, A. E., de Grijs, R., Rubele, S., et al. 2015, MNRAS, 450, 552

Pieres, A., Santiago, B. X., Drlica-Wagner, A., et al. 2017, MNRAS, 468, 1349 Putman, M. E., Gibson, B. K., Staveley-Smith, L., et al. 1998, Nature, 394, 752

Santos, J. F. C., Maia, F. F. S., Dias, B., et al. 2020, MNRAS, 498, 205

Souza, S. O., Kerber, L. O., Barbuy, B., et al. 2020, ApJ, 890, 38

Tokovinin, A., Cantarutti, R., Tighe, R., et al. 2016, PASP, 128

van der Marel, R. P., \& Cioni, M.-R. L. 2001, AJ, 122, 1807

van der Marel, R. P., \& Kallivayalil, N. 2014, ApJ, 781, 121

van der Marel, R. P., Alves, D. R., Hardy, E., \& Suntzeff, N. B. 2002, AJ, 124, 2639

Vasiliev, E. 2018, MNRAS, 481, L100

Zaritsky, D., Harris, J., Thompson, I. B., Grebel, E. K., \& Massey, P. 2002, AJ, 123,855

Zivick, P., Kallivayalil, N., van der Marel, R. P., et al. 2018, ApJ, 864, 55 


\section{Appendix A: Supporting material}

\section{A.1. Isochrone fitting and spectroscopic membership}

Here, we present the CMD isochrone fitting and spectroscopic membership selection.

\section{A.2. Vector-point diagrams from Gaia EDR3}

We present the vector-point diagram (VPD) showing the position of the SMC, Bridge, and all selected cluster stars in Fig. A.3. In all cases, an initial quality filter was applied (see Vasiliev 2018) selecting only stars with $\sigma_{\mu_{\alpha}}<0.2 \mathrm{mas} \mathrm{yr}^{-1}, \sigma_{\mu_{\delta}}<0.2 \mathrm{mas} \mathrm{yr}^{-1}$, and $\pi<3 \sigma_{\pi}$, that is to say a parallax consistent with zero. These criteria resulted in a single star for four clusters; therefore, we relaxed the constraint on proper motions errors from 0.2

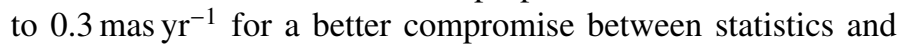
uncertainties. Only one star of B 168 lies outside the plot area and it was considered an outlier. The locus of the SMC is represented by a sample of stars located within $0.5^{\circ}$ from its optical centre. The locus of the Bridge is represented by all good-quality stars within $0.5^{\circ}$ around the cluster BS225 position, which is a random Bridge cluster far away from the SMC centre (Bica et al. 2015). The relative density of stars between the SMC and Bridge was optimised for best visualisation of their positions in the VPD only. The membership selection of stars for each cluster does not use proper motion information, only RVs and metallicities as described above. The stars indicated in Fig. A.3 are those goodquality stars from Gaia that match the selected member stars for each cluster. The proper motions of each cluster is the weighted average of the selected Gaia stars. The systematic uncertainty of $\sigma_{\mu}=0.01 \mathrm{mas} \mathrm{yr}^{-1}$ given by Gaia Collaboration (2021b) is negligible in comparison with the uncertainties reported in Table 1. Looking at Fig. A.3, we confirm that the Bridge clusters are pointing towards the $\mathrm{LMC}$, which is consistent with the Bridge,
Table A.1. Mean parameters adopted for the SMC and LMC.

\begin{tabular}{lcrrr}
\hline \hline \multicolumn{1}{c}{ Param. } & Unit & \multicolumn{1}{c}{ SMC } & \multicolumn{1}{c}{ LMC } & Ref. \\
\hline$\alpha(J 2000)$ & hh:mm:ss & $00: 53: 45$ & $05: 19: 31$ & 1,2 \\
$\delta(J 2000)$ & dd:mm:ss & $-72: 49: 43$ & $-69: 35: 34$ & 1,2 \\
$(\mathrm{~m}-\mathrm{M})$ & $\mathrm{mag}$ & $18.96 \pm 0.02$ & $18.49 \pm 0.09$ & 3,4 \\
$\mathrm{RV}_{\text {helio }}$ & $\mathrm{km} \mathrm{s}^{-1}$ & $149.6 \pm 0.8$ & $262.2 \pm 3.4$ & 5,6 \\
$\mu_{\alpha} \cdot \cos (\delta)$ & $\mathrm{mas} \mathrm{yr}^{-1}$ & $0.721 \pm 0.024$ & $1.910 \pm 0.020$ & 5,7 \\
$\mu_{\delta}$ & $\mathrm{mas} \mathrm{yr}^{-1}$ & $-1.222 \pm 0.018$ & $0.229 \pm 0.047$ & 5,7 \\
\hline
\end{tabular}

References. 1. Crowl et al. (2001); 2. van der Marel \& Kallivayalil (2014); 3. de Grijs \& Bono (2015); 4. de Grijs et al. (2014); 5. De Leo et al. (2020); 6. van der Marel et al. (2002); 7. Kallivayalil et al. (2013).

whereas the Counter-Bridge cluster is moving in the opposite direction.

\section{A.3. $3 D$ view of the SMC clusters}

In order to provide an additional visualisation of the results, we calculated the Cartesian coordinates of the clusters in a reference system, with an origin at the LMC, the $z$-axis pointing towards us, the $y$-axis towards the north, and the $x$-axis towards the west. We applied the Eqs. (1)-(3), and (5) from van der Marel \& Cioni (2001). The velocity vectors in this Cartesian system were calculated using Eqs. (3), and (6)-(8) from van der Marel et al. (2002). We also calculated the velocity vectors for the mean motion of the SMC and subtracted its mean position and velocity from all clusters and the LMC to finally produce Fig. A.4 with positions and velocities relative to the SMC. The adopted mean position and velocities of the SMC and LMC are given in Table A.1. It is very clear that cluster IC 1708 and the Bridge clusters are moving in opposite directions, roughly aligned with the SMC-LMC direction, as it can also be seen in Fig. A.3. 
B. Dias et al.: The VISCACHA survey. III.
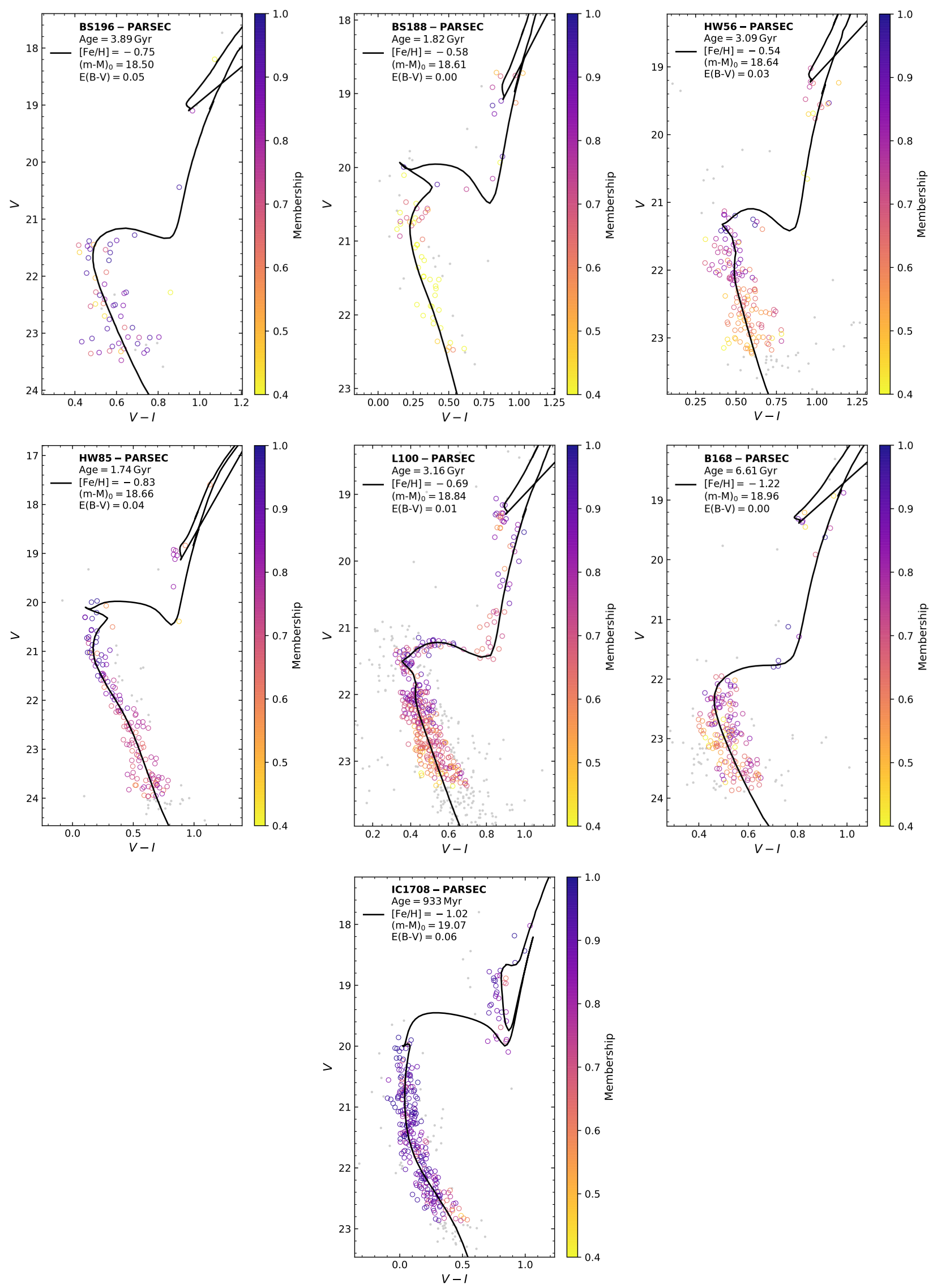

Fig. A.1. Statistically decontaminated CMD with the best isochrone fitting for each cluster analysed here, using the SIRIUS code. Grey dots represent field stars, whereas the circles are probable cluster member stars, colour-coded by their membership probability. 

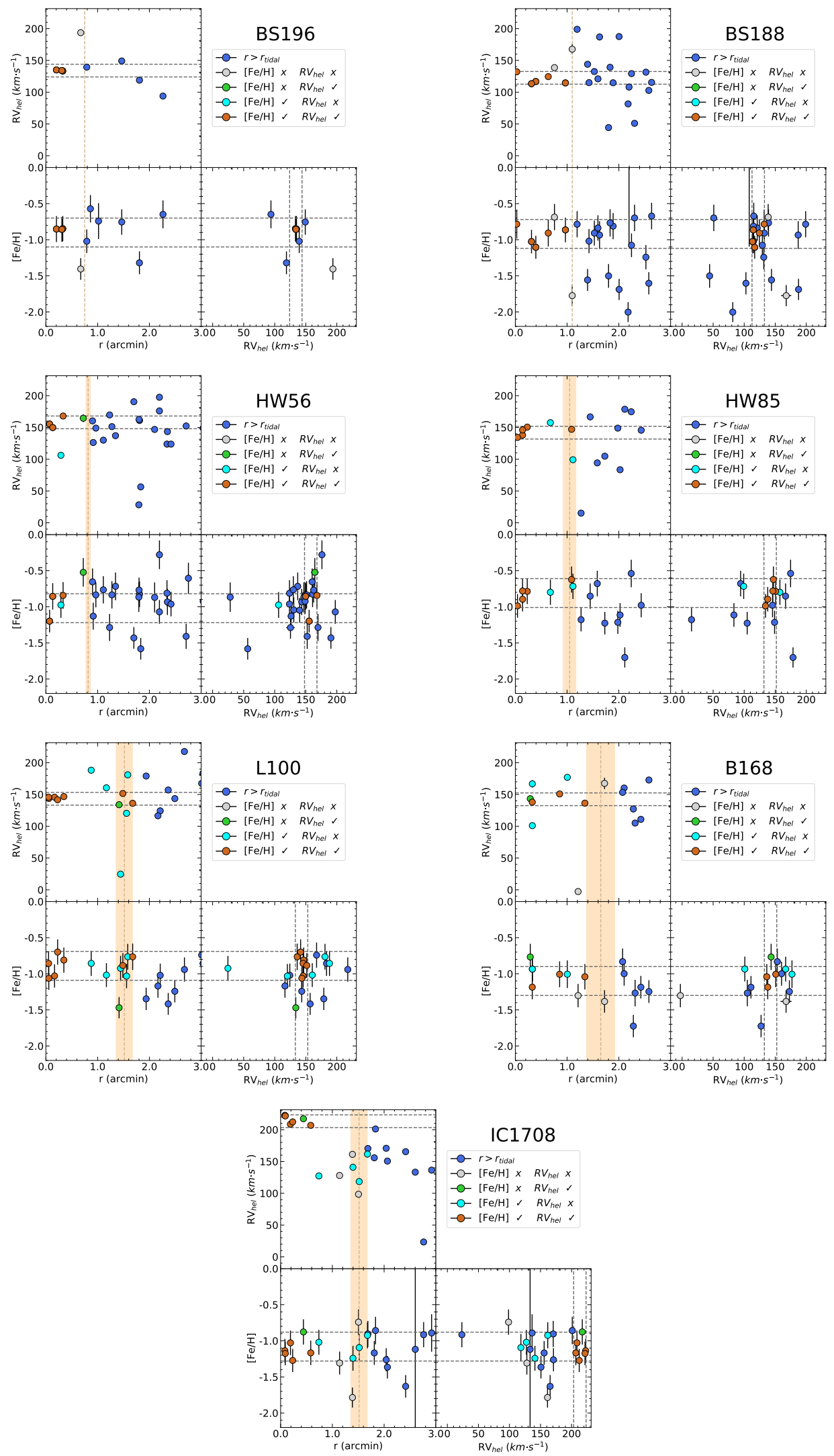

Fig. A.2. Membership selection of cluster stars with spectroscopic information. The shaded area marks the cluster tidal radius $\pm 1 \sigma$ from Santos et al. (2020), otherwise only a line represents twice the visual radius by Bica et al. (2020). The limits in $[\mathrm{Fe} / \mathrm{H}]$ and $\mathrm{RV}$ hel $\operatorname{are} 0.2 \mathrm{dex}$ and $10 \mathrm{~km} \mathrm{~s}^{-1}$ around the group of innermost stars. 

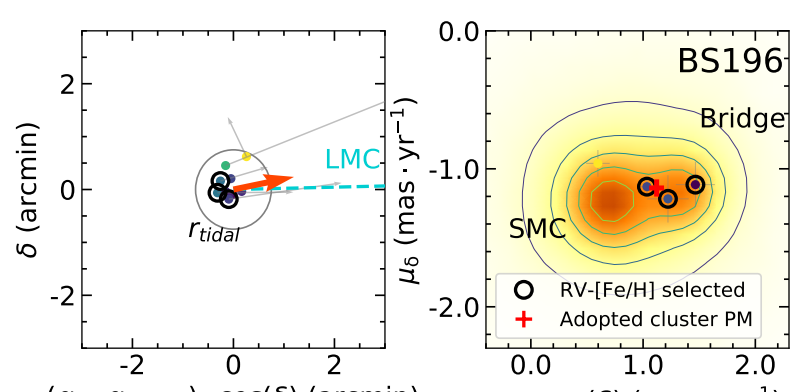

$\left(\alpha-\alpha_{\text {cluster }}\right) \cdot \cos (\delta)(\operatorname{arcmin})$

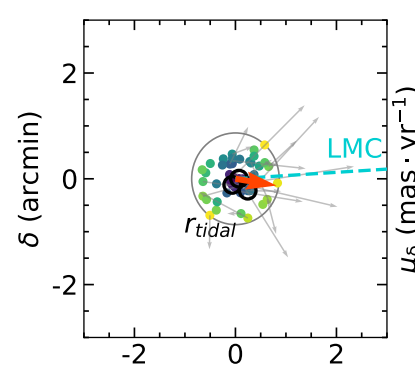

$\left(\alpha-\alpha_{\text {cluster }}\right) \cdot \cos (\delta)(\operatorname{arcmin})$

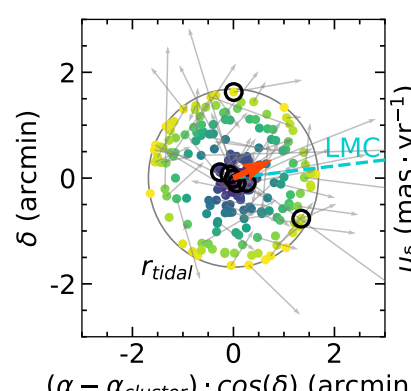

$\left(\alpha-\alpha_{\text {cluster }}\right) \cdot \cos (\delta)(\operatorname{arcmin})$

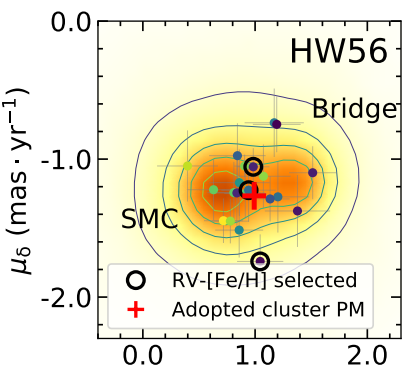

$\mu_{\alpha} \cdot \cos (\delta)\left(\right.$ mas $\left.\cdot y^{-1}\right)$

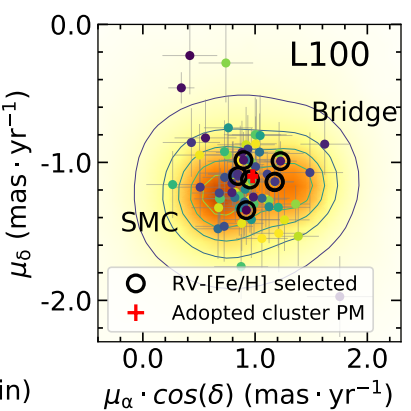

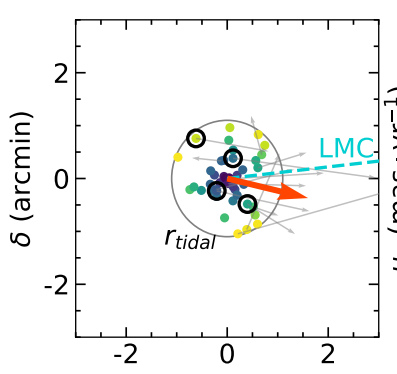

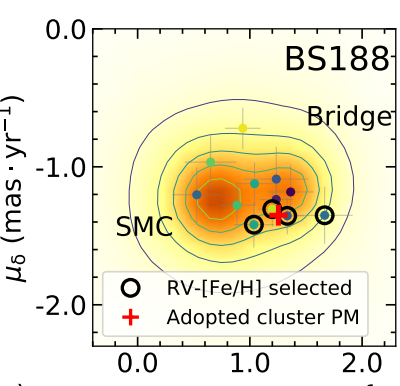

$\left(\alpha-\alpha_{\text {cluster }}\right) \cdot \cos (\delta)(\operatorname{arcmin})$

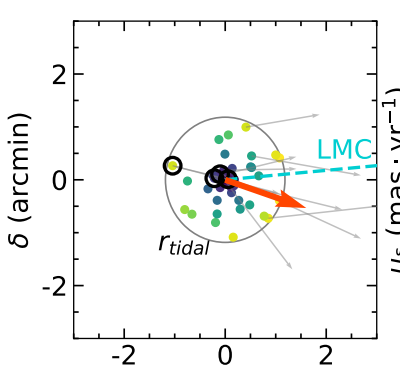

$\left(\alpha-\alpha_{\text {cluster }}\right) \cdot \cos (\delta)(\operatorname{arcmin})$

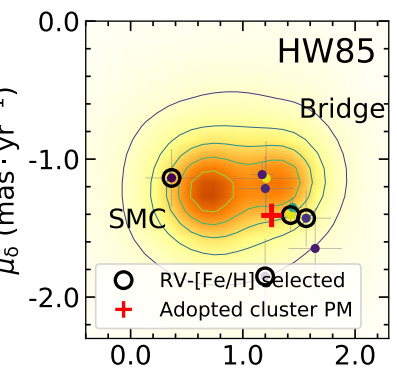

$\mu_{\alpha} \cdot \cos (\delta)\left(\right.$ mas $\left.\cdot \mathrm{yr}^{-1}\right)$
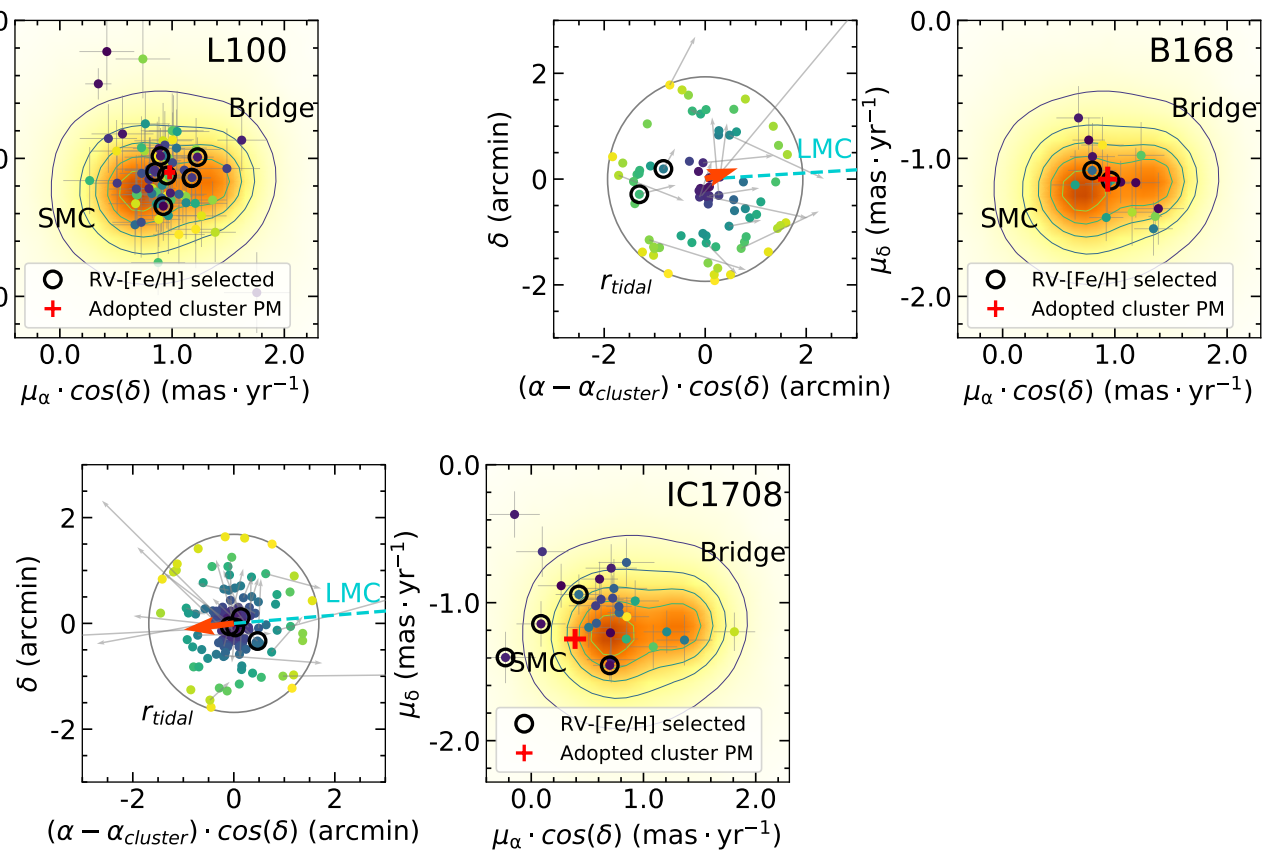

Fig. A.3. Vector-point diagram from Gaia EDR3 proper motions for the seven clusters. Left panel: on sky distribution of all Gaia stars within the tidal radius of the cluster. Colours indicate the distance from the cluster centre. Grey arrows are Gaia EDR3 good-quality proper motions subtracted from the SMC mean proper motion. The red thick arrow is the average of the selected member stars highlighted with black circles. The direction of the LMC is indicated by the turquoise line. Right panel: the background density plot represents the locus of the SMC and Bridge based on a sample of stars (see text for details). The points and their error bars are the equivalent from the left panel. 
A\&A 647, L9 (2021)

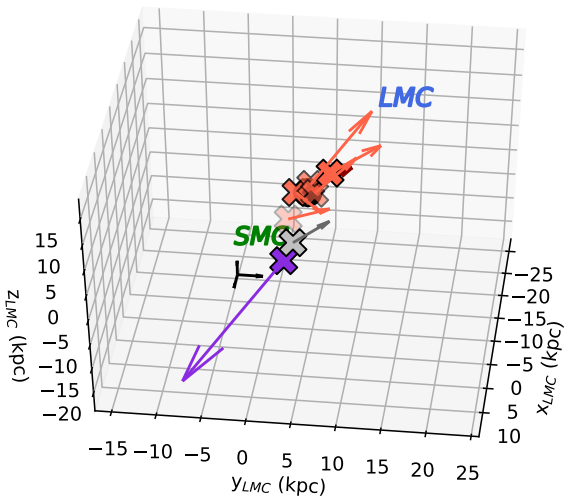

Fig. A.4. Phase-space vectors of the seven clusters analysed in this work in a 3D Cartesian system centred at the SMC as described in the text. Arrows are the velocities relative to the SMC mean velocity. Colours and symbols are the same as in Fig. 2. The average velocity of the five Bridge clusters is shown in dark red. The black arrows are a reference scale representing $10 \mathrm{~km} \mathrm{~s}^{-1}$. A movie is available online. 